\title{
Weakly Noncollapsed RCD Spaces with Upper Curvature Bounds
}

https://doi.org/10.1515/agms-2019-0010

Received June 27, 2019; accepted September 22, 2019.

Abstract: We show that if a $C D(K, n)$ space $\left(X, d, f \mathcal{H}^{n}\right)$ with $n \geq 2$ has curvature bounded above by $\kappa$ in the sense of Alexandrov then $f$ is constant.

Keywords: Riemannian curvature-dimension condition, upper curvature bound, Alexandrov space, optimal transport

MSC: Primary 53C20, 53C21

\section{Introduction}

In [16] Gigli and De Philippis introduced the following notion of a noncollapsed $R C D(K, n)$ space. An $R C D(K, n)$ space $(X, d, m)$ is noncollapsed if $n$ is a natural number and $m=\mathcal{H}^{n}$. A similar notion was considered by Kitabeppu in [23].

Noncollapsed $R C D(K, n)$ spaces give a natural intrinsic generalization of noncollapsing limits of manifolds with lower Ricci curvature bounds which are noncollapsed in the above sense by work of CheegerColding [13].

In [16] Gigli and De Philippis also considered the following a-priori weaker notion. An $\operatorname{RCD}(K, n)$ space $(X, d, m)$ is weakly noncollapsed if $n$ is a natural number and $m \ll \mathcal{H}^{n}$. Gigli and De Philippis gave several equivalent characterizations of weakly noncollapsed $R C D(K, n)$ spaces and studied their properties. By work of Mondino-Naber [31], Kell-Mondino [25] and Brué-Semola [11] it follows that an $R C D(K, n)$ space is weakly noncollapsed iff $\mathcal{R}_{n} \neq \emptyset$ where $\mathcal{R}_{n}$ is the rectifiable set of $n$-regular points in $X$.

It is well-known that if $(X, d, m)=\left(M^{n}, g, e^{-f} d \operatorname{vol}_{g}\right)$ where $\left(M^{n}, g\right)$ is a smooth $n$-dimensional Riemannian manifold and $f$ is a smooth function on $M$ then $(X, d, m)$ is $R C D(K, n)$ iff $f=$ const. More precisely, the classical Bakry-Emery condition $B E(K, N), K \in \mathbb{R}$ and $N \geq n$, for a (compact) smooth metric measure space $\left(M^{n}, g, e^{-f} d \operatorname{vol}_{g}\right), f \in C^{\infty}(M)$, is

$$
\frac{1}{2} L|\nabla u|_{g}^{2} \geq\langle\nabla L u, \nabla u\rangle_{g}+\frac{1}{N}(L u)^{2}+K|\nabla u|_{g}^{2}, \quad \forall u \in C^{\infty}(M),
$$

where $L=\Delta-\nabla f$. In [8, Proposition 6.2] Bakry shows that $B E(K, N)$ holds if and only if

$$
\nabla f \otimes \nabla f \leq(N-n)\left(\text { ric }_{g}+\nabla^{2} f-K g\right) .
$$

In particular, if $N=n$, then $f$ is locally constant.

On the other hand, in $[5,6,18]$ it was proven that a metric measure space $(X, d, m)$ satisfies $R C D(K, N)$ if and only if the corresponding Cheeger energy satifies a weak version of $B E(K, N)$ that is equivalent to the classical version for $\left(M, g, e^{-f} \operatorname{vol}_{g}\right)$ from above. 
In [16] Gigli and De Philippis conjectured that a weakly noncollapsed $R C D(K, n)$ space is already noncollapsed up to rescaling of the measure by a constant. Our main result is that this conjecture holds if a weakly noncollapsed space has curvature bounded above in the sense of Alexandrov.

Theorem 1.1. Let $n \geq 2$ and let $\left(X, d, f \mathcal{H}^{n}\right)$ (where $f$ is $L_{\text {loc }}^{1}$ with respect to $\mathcal{H}^{n}$ and $\operatorname{supp}\left(f \mathcal{H}^{n}\right)=X$ ) be a complete metric measure space which is $C B A(\kappa)$ (has curvature bounded above by $\kappa$ in the sense of Alexandrov) and satisfies $C D(K, n)$. Then $f=$ const $^{1}$.

Since smooth Riemannian manifolds locally have curvature bounded above this immediately implies

Corollary 1.2. Let $\left(M^{n}, g\right)$ be a smooth Riemannian manifold and suppose $\left(M^{n}, g, f \mathcal{H}{ }^{n}\right)$ is $C D(K, n)$ where $K$ is finite and $f \geq 0$ is $L_{\text {loc }}^{1}$ with respect to $\mathcal{H}^{n}$ and $\operatorname{supp}\left(f \mathcal{H}^{n}\right)=M$. Then $f=$ const.

As was mentioned above, this corollary was well-known in case of smooth $f$ but was not known in case of general locally integrable $f$.

In [24] it was shown that if a space $(X, d, m)$ is $C D(K, n)$ and has curvature bounded above then $X$ is $R C D(K, n)$ and if in addition $m=\mathcal{H}^{n}$ then $X$ is Alexandrov with two sided curvature bounds. Combined with Theorem 1.1 this implies that the same remains true if the assumption on the measure is weakened to $m \ll \mathcal{H}^{n}$.

Corollary 1.3. Let $n \geq 2$ and let $\left(X, d, f \mathcal{H}^{n}\right)$ where $f$ is $L_{\text {loc }}^{1}$ with respect to $\mathcal{H}^{n}$ and $\operatorname{supp}\left(f \mathcal{H}^{n}\right)=X$ be a complete metric measure space which is $C B A(\kappa)$ (has curvature bounded above by $\kappa$ in the sense of Alexandrov) and satisfies $C D(K, n)$. Then $X$ is $R C D(K, n), f=$ const, $k(n-1) \geq K$, and $(X, d)$ is an Alexandrov space of curvature bounded below by $K-\kappa(n-2)$.

Remark 1.4. Note that since a space $\left(X, d, f \mathcal{H}^{n}\right)$ satisfying the assumptions of Theorem 1.1 is automatically $R C D(K, n)$, as was remarked in [16] it follows from the results of [25] that $n$ must be an integer.

Bakry's proof for smooth manifolds does not easily generalize to a non-smooth context. But let us describe a strategy that does generalize to $R C D+C A T$ spaces.

Assume that $(X, d)$ is induced by a smooth manifold $\left(M^{n}, g\right)$ and the density function $f$ is smooth and positive such that $(X, d, f m)$ satisfies $R C D(K, n)$. Then, by integration by parts on $(M, g)$ the induced Laplace operator $L$ is given by

$$
L u=\Delta u-\langle\nabla \log f, \nabla u\rangle, u \in C^{\infty}(M),
$$

where $\Delta u$ is the classical Laplace-Beltrami operator of $(M, g)$ for smooth functions. By a recent result of Han one has for any $R C D(K, n)$ space that the operator $L$ is equal to the trace of Gigli's Hessian [20] on the set of $n$-regular points $\mathcal{R}_{n}$. Hence, after one identifies the trace of Gigli's Hessian with the Laplace-Beltrami operator $\Delta$ of $M$ (what is true on $\left(M^{n}, g\right)$ ), one obtains immediately that $\nabla \log f=0$. If $M$ is connected, this yields the claim.

The advantage of this approach is that it does not involve the Ricci curvature tensor and in non-smooth context one might follow the same strategy. However, we have to overcome several difficulties that arise from the non-smoothness of the density function $f$ and of the space $(X, d, m)$.

In particular, we apply the recently developed $D C$ calculus by Lytchak-Nagano for spaces with upper curvature bounds to show that on the regular part of $X$ the Laplace operator with respect to $\mathcal{H}^{n}$ is equal to the trace of the Hessian. By [24] the combination of CD and CAT condition implies that $f^{1 / N}$ is locally semiconcave and hence $f$ is locally Lipschitz on the regular part of $X$. This allows us to generalize the above argument for smooth Riemannian manifolds to the general case.

1 Here and in all applications by $f=$ const we mean $f=$ const a.e. with respect to $\mathcal{H}^{n}$. 
In section 2 we provide necessary preliminaries. We present the setting of $R C D$ spaces and the calculus for them. We state important results by Mondino-Cavalletti (Theorem 2.4), Han (Theorem 2.11) and Gigli (Theorem 2.7, Proposition 2.9). We also give a brief introduction to the calculus of $B V$ and $D C$ functions for spaces with upper curvature bounds.

In section 3 we develop a structure theory for general $R C D+C A T$ spaces where we adapt the $D C$ calculus of Lytchak-Nagano [27]. This might be of independent interest.

Finally, in section 4 we prove our main theorem following the above idea.

\section{Preliminaries}

\subsection{Curvature-dimension condition}

A metric measure space is a triple $(X, d, m)$ where $(X, d)$ is a complete and separable metric space and $m$ is a locally finite measure.

$\mathcal{P}^{2}(X)$ denotes the set of Borel probability measures $\mu$ on $(X, d)$ such that $\int_{X} d\left(x_{0}, x\right)^{2} d \mu(x)<\infty$ for some $x_{0} \in X$ equipped with $L^{2}$-Wasserstein distance $W_{2}$. The sub-space of $m$-absolutely continuous probability measures in $\mathcal{P}^{2}(X)$ is denoted by $\mathcal{P}^{2}(X, m)$.

The $N$-Renyi entropy is

$$
S_{N}(\cdot \mid m): \mathcal{P}_{b}^{2}(X) \rightarrow(-\infty, 0], S_{N}(\mu \mid m)=-\int \rho^{1-\frac{1}{N}} d m \text { if } \mu=\rho m \text {, and } 0 \text { otherwise. }
$$

Note that $\rho m \in \mathcal{P}^{2}(X, m)$ implies $\rho \in L^{1}(m)$. The functional $S_{N}$ is lower semi-continuous, and $S_{N}(\mu) \geq$ $-m(\operatorname{supp} \mu)^{\frac{1}{N}}$ by Jensen's inequality.

For $\kappa \in \mathbb{R}$ we define

$$
\cos _{\kappa}(x)=\left\{\begin{array}{ll}
\cosh (\sqrt{|\kappa|} x) & \text { if } \kappa<0 \\
1 & \text { if } \kappa=0 \\
\cos (\sqrt{\kappa} x) & \text { if } \kappa>0
\end{array} \quad \sin _{\kappa}(x)= \begin{cases}\frac{\sinh (\sqrt{|\kappa|} x)}{\sqrt{|\kappa|}} & \text { if } \kappa<0 \\
x & \text { if } \kappa=0 \\
\frac{\sin (\sqrt{\kappa} x)}{\sqrt{\kappa}} & \text { if } \kappa>0\end{cases}\right.
$$

Let $\pi_{\kappa}$ be the diameter of a simply connected space form $\mathbb{S}_{\kappa}^{2}$ of constant curvature $\kappa$, i.e.

$$
\pi_{\kappa}= \begin{cases}\infty & \text { if } \kappa \leq 0 \\ \frac{\pi}{\sqrt{\kappa}} & \text { if } \kappa>0\end{cases}
$$

For $K \in \mathbb{R}, N \in(0, \infty)$ and $\theta \geq 0$ we define the distortion coefficient as

$$
t \in[0,1] \mapsto \sigma_{K, N}^{(t)}(\theta)= \begin{cases}\frac{\sin _{K / N}(t \theta)}{\sin _{K / N}(\theta)} & \text { if } \theta \in\left[0, \pi_{K / N}\right), \\ \infty & \text { otherwise. }\end{cases}
$$

Note that $\sigma_{K, N}^{(t)}(0)=t$. For $K \in \mathbb{R}, N \in[1, \infty)$ and $\theta \geq 0$ the modified distortion coefficient is

$$
t \in[0,1] \mapsto \tau_{K, N}^{(t)}(\theta)= \begin{cases}\theta \cdot \infty & \text { if } K>0 \text { and } N=1, \\ t^{\frac{1}{N}}\left[\sigma_{K, N-1}^{(t)}(\theta)\right]^{1-\frac{1}{N}} & \text { otherwise. }\end{cases}
$$

Definition 2.1 ([29, 35]). We say $(X, d, m)$ satisfies the curvature-dimension condition $C D(K, N)$ for $K \in \mathbb{R}$ and $N \in[1, \infty)$ if for every $\mu_{0}, \mu_{1} \in \mathcal{P}_{b}^{2}(X, m)$ there exists an $L^{2}$-Wasserstein geodesic $\left(\mu_{t}\right)_{t \in[0,1]}$ and an optimal coupling $\pi$ between $\mu_{0}$ and $\mu_{1}$ such that

$$
S_{N}\left(\mu_{t} \mid m\right) \leq-\int\left[\tau_{K, N}^{(1-t)}(d(x, y)) \rho_{0}(x)^{-\frac{1}{N}}+\tau_{K, N}^{(t)}(d(x, y)) \rho_{1}(y)^{-\frac{1}{N}}\right] d \pi(x, y)
$$

where $\mu_{i}=\rho_{i} d m, i=0,1$. 
Remark 2.2. If $(X, d, m)$ is complete and satisfies the condition $C D(K, N)$ for $N<\infty$, then (supp $m, d)$ is a geodesic space and ( $\operatorname{supp} m, d, m)$ is $C D(K, N)$.

In the following we always assume that supp $m=X$.

Remark 2.3. For the variants $C D^{\star}(K, N)$ and $C D^{e}(K, N)$ of the curvature-dimension condition we refer to [12, $18]$.

\subsection{Calculus on metric measure spaces}

For further details about this section we refer to $[2-4,19]$.

Let $(X, d, m)$ be a metric measure space, and let $\operatorname{Lip}(X)$ be the space of Lipschitz functions. For $f \in \operatorname{Lip}(X)$ the local slope is

$$
\operatorname{Lip}(f)(x)=\limsup _{y \rightarrow x} \frac{|f(x)-f(y)|}{d(x, y)}, x \in X .
$$

If $f \in L^{2}(m)$, a function $g \in L^{2}(m)$ is called relaxed gradient if there exists sequence of Lipschitz functions $f_{n}$ which $L^{2}$-converges to $f$, and there exists $h$ such that $\operatorname{Lip} f_{n}$ weakly converges to $h$ in $L^{2}(m)$ and $h \leq g m$ a.e.. $g \in L^{2}(m)$ is called the minimal relaxed gradient of $f$ and denoted by $|\nabla f|$ if it is a relaxed gradient and minimal w.r.t. the $L^{2}$-norm amongst all relaxed gradients. The space of $L^{2}$-Sobolev functions is then

$$
W^{1,2}(X):=D\left(\mathrm{Ch}^{X}\right):=\left\{f \in L^{2}(m): \int|\nabla f|^{2} d m<\infty\right\} .
$$

$W^{1,2}(X)$ equipped with the norm $\|f\|_{W^{1,2}(X)}^{2}=\|f\|_{L^{2}}^{2}+\||\nabla f|\|_{L^{2}}^{2}$ is a Banach space. If $W^{1,2}(X)$ is a Hilbert space, we say $(X, d, m)$ is infinitesimally Hilbertian. In this case we can define

$$
(f, g) \in W^{1,2}(X)^{2} \mapsto\langle\nabla f, \nabla g\rangle:=\frac{1}{4}|\nabla(f+g)|^{2}-\frac{1}{4}|\nabla(f-g)|^{2} \in L^{1}(m) .
$$

Assuming $X$ is locally compact, if $U$ is an open subset of $X$, we say $f \in W^{1,2}(X)$ is in the domain $D(\Delta, U)$ of the measure valued Laplace $\Delta$ on $U$ if there exists a signed Radon functional $\Delta f$ on the set of Lipschitz functions $g$ with bounded support in $U$ such that

$$
\int\langle\nabla g, \nabla f\rangle d m=-\int g d \Delta f
$$

If $U=X$ and $\Delta f=[\boldsymbol{\Delta} f]_{a c} m$ with $[\Delta f]_{a c} \in L^{2}(m)$, we write $[\Delta f]_{a c}=: \Delta f$ and $D(\boldsymbol{\Delta}, X)=D_{L^{2}(m)}(\Delta) . \mu_{a c}$ denotes the $m$-absolutely continuous part in the Lebesgue decomposition of a Borel measure $\mu$. If $\mathbb{V}$ is any subspace of $L^{2}(m)$ and $f \in D_{L^{2}(m)}(\Delta)$ with $\Delta f \in \mathbb{V}$, we write $f \in D_{\mathbb{V}}(\Delta)$.

Theorem 2.4 (Cavalletti-Mondino, [15]). Let $(X, d, m)$ be an essentially non-branching $C D(K, N)$ space for some $K \in \mathbb{R}$ and $N>1$. For $p \in X$ consider $d_{p}=d(p, \cdot)$ and the associated disintegration $m=\left.\int_{Q} h_{\alpha} \mathcal{H}^{1}\right|_{X_{\alpha}} q(d \alpha)$.

Then $d_{p} \in D(\Delta, X \backslash\{p\})$ and $\left.\Delta d_{p}\right|_{X \backslash\{p\}}$ has the following representation formula:

$$
\left.\Delta d_{p}\right|_{X \backslash\{p\}}=-\left(\log h_{\alpha}\right)^{\prime} m-\int_{Q} h_{\alpha} \delta_{a\left(X_{\alpha}\right)} q(d \alpha) .
$$

Moreover

$$
\left.\Delta d_{p}\right|_{X \backslash\{p\}} \leq(N-1) \frac{\sin _{K /(N-1)}^{\prime}\left(d_{p}(x)\right)}{\sin _{K /(N-1)}\left(d_{p}(x)\right)} m \&\left[\left.\Delta d_{p}\right|_{X \backslash\{p\}}\right]^{r e g} \geq-(N-1) \frac{\sin _{K /(N-1)}^{\prime}\left(d_{p}(x)\right)}{\sin _{K /(N-1)}\left(d_{p}(x)\right)} m .
$$

Remark 2.5. The sets $X_{\alpha}$ in the previous disintegration are geodesic segments $\left[a\left(X_{\alpha}\right), p\right]$ with initial point $a\left(X_{\alpha}\right)$ and endpoint $p$. In particular, the set of points $q \in X$ such that there exists a geodesic connecting $p$ and $q$ that is extendible beyond $q$, is a set of full measure. 
Definition 2.6. A metric measure space $(X, d, m)$ satisfies the Riemannian curvature-dimension condition $R C D(K, N)$ for $K \in \mathbb{R}$ and $N \in[1, \infty]$ if it satisfies a curvature-dimension conditions $C D(K, N)$ and is infinitesimally Hilbertian.

The previous definition was first proposed in [4] for $N=\infty$. For a brief overview on the historical development in the case $N<\infty$ we refer to [24, Remark 2.15].

In [20] Gigli introduced a notion of Hess $f$ in the context of $R C D$ spaces. Hess $f$ is tensorial and defined for $f \in W^{2,2}(X)$ that is the second order Sobolev space. An important property of $W^{2,2}(X)$ that we will need in the following is

Theorem 2.7 (Corollary 3.3.9 in [20], [34]). $D_{L^{2}(m)}(\Delta) \subset W^{2,2}(X)$.

Remark 2.8. The closure of $D_{L^{2}(m)}(\Delta)$ in $W^{2,2}(X)$ is denoted $H^{2,2}(X)$ [20, Proposition 3.3.18].

The next proposition [20, Proposition 3.3.22 i)] allows to compute Hess $f$ explicitely.

Proposition 2.9. Let $f, g_{1}, g_{2} \in H^{2,2}(X)$. Then $\left\langle\nabla f, \nabla g_{i}\right\rangle \in W^{1,2}(X)$, and

$$
2 \operatorname{Hess} f\left(\nabla g_{1}, \nabla g_{2}\right)=\left\langle\nabla g_{1}, \nabla\left\langle\nabla f, \nabla g_{2}\right\rangle\right\rangle+\left\langle\nabla g_{2}, \nabla\left\langle\nabla f, \nabla g_{1}\right\rangle\right\rangle+\left\langle\nabla f, \nabla\left\langle\nabla g_{1}, \nabla g_{2}\right\rangle\right\rangle
$$

holds $m$-a.e. where the two sides in this expression are well-defined in $L^{0}(m)$.

Theorem 2.10 ([11]). Let $(X, d, m)$ be a metric measure space satisfying $R C D(K, N)$ with $N<\infty$. Then, there exist $n \in \mathbb{N}$ and such that the set of n-regular points $\mathcal{R}_{n}$ has full measure.

Theorem 2.11 ([22]). Let $(X, d, m)$ be as in the previous theorem. If $N=n \in \mathbb{N}$, then for any $f \in \mathbb{D}_{\infty}$ we have that $\Delta f=\operatorname{tr}$ Hess $f$ m-a.e. . More precisely, if $B \subset \mathcal{R}_{n}$ is a set of finite measure and $\left(e_{i}\right)_{i=1, \ldots, n}$ is a unit orthogonal basis on $B$, then

$$
\left.\Delta f\right|_{B}=\sum_{i=1}^{n} \operatorname{Hess} f\left(e_{i}, e_{i}\right) 1_{B}=:\left.[\operatorname{tr} \operatorname{Hess} f]\right|_{B} .
$$

Corollary 2.12. Let $(X, d, m)$ be a metric measure space as before. If $f \in D_{L^{2}(m)}(\Delta)$, we have that $\Delta f=\operatorname{tr}$ Hess $f$ $m$-a.e. in the sense of the previous theorem.

\subsection{Spaces with upper curvature bounds}

We will assume familiarity with the notion of $\operatorname{CAT}(\kappa)$ spaces. We refer to $[9,10]$ or $[24]$ for the basics of the theory.

Definition 2.13. Given a point $p$ in a $C A T(\kappa)$ space $X$ we say that two unit speed geodesics starting at $p$ define the same direction if the angle between them is zero. This is an equivalence relation by the triangle inequality for angles and the angle induces a metric on the set $S_{p}^{g}(X)$ of equivalence classes. The metric completion $\Sigma_{p}^{g} X$ of $S_{p}^{g} X$ is called the space of geodesic directions at $p$. The Euclidean cone $C\left(\Sigma_{p}^{g} X\right)$ is called the geodesic tangent cone at $p$ and will be denoted by $T_{p}^{g} X$.

The following theorem is due to Nikolaev [10, Theorem 3.19]:

Theorem 2.14. $T_{p}^{g} X$ is $C A T(0)$ and $\Sigma_{p}^{g} X$ is $C A T(1)$.

Note that this theorem in particular implies that $T_{p}^{g} X$ is a geodesic metric space which is not obvious from the definition. More precisely, it means that each path component of $\Sigma_{p}^{g} X$ is $C A T(1)$ (and hence geodesic) and the distance between points in different components is $\pi$. Note however, that $\Sigma_{p}^{g} X$ itself need not be path connected. 


\section{$2.4 B V$ functions and $D C$ calculus}

Recall that a function $g: V \subset \mathbb{R}^{n} \rightarrow \mathbb{R}$ of bounded variation $(B V)$ admits a derivative in the distributional sense $\left[17\right.$, Theorem 5.1] that is a signed vector valued Radon measure $[D g]=\left(\frac{\partial g}{\partial x_{1}}, \ldots, \frac{\partial g}{\partial x_{n}}\right)=[D g]_{a c}+[D g]_{s}$. Moreover, if $g$ is $B V$, then it is $L^{1}$-differentiable [17, Theorem 6.1] a.e. with $L^{1}$-derivative [Dg $]_{a c}$, and approximately differentiable a.e. [17, Theorem 6.4] with approximate derivative $D^{a p} g=\left(\frac{\partial^{a p} g}{\partial x_{1}}, \ldots, \frac{\partial^{a p} g}{\partial x_{n}}\right)$ that coincides almost everywhere with $[D g]_{a c}$. The set of $B V$ functions $B V(V)$ on $V$ is closed under addition and multiplication [32, Section 4]. We'll call $B V$ functions $B V_{0}$ if they are continuous.

Remark 2.15. In [32] and [1] $B V$ functions are called $B V_{0}$ if they are continuous away from an $\mathcal{H}^{n-1}$-negligible set. However, for the purposes of the present paper it will be more convenient to work with the more restrictive definition above.

Then for $f, g \in B V_{0}(V)$ we have

$$
\frac{\partial f g}{\partial x_{i}}=\frac{\partial f}{\partial x_{i}} g+f \frac{\partial g}{\partial x_{j}}
$$

as signed Radon measures [32, Section 4, Lemma]. By taking the $\mathcal{L}^{n}$-absolutely continuous part of this equality it follows that (2.3) also holds a.e. in the sense of approximate derivatives. In fact, it holds at all points of approximate differentiability of $f$ and $g$. This easily follows by a minor variation of the standard proof that $d(f g)=f d g+g d f$ for differentiable functions.

A function $f: V \subset \mathbb{R}^{n} \rightarrow \mathbb{R}$ is called a $D C$ function if in a small neighborhood of each point $x \in V$ one can write $f$ as a difference of two semi-convex functions. The set of $D C$ functions on $V$ is denoted by $D C(V)$ and contains the class $C^{1,1}(V)$. The space $D C(V)$ is closed under addition and multiplication. The first partial derivatives $\frac{\partial f}{\partial x_{i}}$ of a $D C$ function $f: V \rightarrow \mathbb{R}$ are $B V$, and hence the second partial derivatives $\frac{\partial}{\partial x_{j}} \frac{\partial f}{\partial x_{i}}$ exist as signed Radon measure that satisfy

[17, Theorem 6.8], and hence

$$
\frac{\partial}{\partial x_{i}} \frac{\partial f}{\partial x_{j}}=\frac{\partial}{\partial x_{j}} \frac{\partial f}{\partial x_{i}}
$$

$$
\frac{\partial^{a p}}{\partial x_{i}} \frac{\partial f}{\partial x_{j}}=\frac{\partial^{a p}}{\partial x_{j}} \frac{\partial f}{\partial x_{i}} \quad \text { a.e. on } V .
$$

A map $F: V \rightarrow \mathbb{R}^{l}, l \in \mathbb{N}$, is called a $D C$ map if each coordinate function $F_{i}$ is $D C$. The composition of two $D C$ maps is again $D C$. A function $f$ on $V$ is called $D C_{0}$ if it's $D C$ and $C^{1}$.

Let $(X, d)$ be a geodesic metric space. A function $f: X \rightarrow \mathbb{R}$ is called a $D C$ function if it can be locally represented as the difference of two Lipschitz semi-convex functions. A map $F: Z \rightarrow Y$ between metric spaces $Z$ and $Y$ that is locally Lipschitz is called a $D C$ map if for each $D C$ function $f$ that is defined on an open set $U \subset Y$ the composition $f \circ F$ is $D C$ on $F^{-1}(U)$. In particular, a map $F: Z \rightarrow \mathbb{R}^{l}$ is $D C$ if and only if its coordinates are $D C$. If $F$ is a bi-Lipschitz homeomorphism and its inverse is $D C$, we say $F$ is a $D C$ isomorphism.

\subsection{DC coordinates in CAT spaces}

The following was developed in [27] based on previous work by Perelman [32].

Assume $(X, d)$ is a CAT space, let $p \in X$ such that there exists an open neighborhood $\hat{U}$ of $p$ that is homeomorphic to $\mathbb{R}^{n}$. It is well known (see e.g. [24, Lemma 3.1] ) that this implies that geodesics in $\hat{U}$ are locally extendible.

Suppose $T_{p}^{g} X \cong \mathbb{R}^{n}$.

Then, there exist $D C$ coordinates near $p$ with respect to which the distance on $\hat{U}$ is induced by a $B V$ Riemannian metric $g$.

More precisely, let $a_{1}, \ldots, a_{n}, b_{1}, \ldots, b_{n}$ be points near $p$ such that $d\left(p, a_{i}\right)=d\left(p, b_{i}\right)=r, p$ is the midpoint of $\left[a_{i}, b_{i}\right]$ and $\angle a_{i} p a_{j}=\pi / 2$ for all $i \neq j$ and all comparison angles $\widetilde{\angle} a_{i} p a_{j}, \widetilde{\angle} a_{i} p b_{j}, \widetilde{\angle} b_{i} p b_{j}$ are sufficiently close to $\pi / 2$ for all $i \neq j$. 
Let $x: \hat{U} \rightarrow \mathbb{R}^{n}$ be given by $x=\left(x_{1}, \ldots, x_{n}\right)=\left(d\left(\cdot, a_{1}\right), \ldots, d\left(\cdot, a_{n}\right)\right)$.

Then by [27, Corollary 11.12] for any sufficiently small $0<\varepsilon<\pi_{k} / 4$ the restriction $\left.x\right|_{B_{2 \varepsilon}(p)}$ is Bilipschitz onto an open subset of $\mathbb{R}^{n}$. Let $U=B_{\varepsilon}(p)$ and $V=x(U)$. By [27, Proposition 14.4] $x: U \rightarrow V$ is a $D C$ equivalence in the sense that $h: U \rightarrow \mathbb{R}$ is $D C$ iff $h \circ x^{-1}$ is $D C$ on $V$.

Further, the distance on $U$ is induced by a $B V$ Riemannian metric $g$ which in $x$ coordinates is given by a 2-tensor $g^{i j}(p)=\cos \alpha_{i j}$ where $\alpha_{i j}$ is the angle at $p$ between geodesics connecting $p$ and $a_{i}$ and $a_{j}$ respectively. By the first variation formula $g^{i j}$ is the derivative of $d\left(a_{i}, y(t)\right)$ at 0 where $y$ is the geodesic with $y(0)=p$ and $y(1)=a_{j}$. Since $d\left(a_{i}, \cdot\right), i=1, \ldots n$, are Lipschitz, $g^{i j}$ is in $L^{\infty}$. We denote $\langle v, w\rangle_{g}(p)=g^{i j}(p) v_{i} w_{j}$ the inner product of $v, w \in \mathbb{R}^{n}$ at $p$. The Riemannian metric $g^{i j}$ induces a distance function $d_{g}$ on $V$ such that $x$ is a metric space isomorphism for $\epsilon>0$ sufficiently small.

If $u$ is a Lipschitz function on $U, u \circ x^{-1}$ is a Lipschitz function on $V$, and therefore differentiable $\mathcal{L}^{n}$-a.e. in $V$ by Rademacher's theorem. Hence, we can define the gradient of $u$ at points of differentiability of $u$ in the usual way as the metric dual of its differential. Then the usual Riemannian formulas hold and $\nabla u=g^{i j} \frac{\partial u}{\partial x_{i}} \frac{\partial}{\partial x_{j}}$ and $|\nabla u|_{g}^{2}=g^{i j} \frac{\partial u}{\partial x_{i}} \frac{\partial u}{\partial x_{j}}$ a.e. .

\section{Structure theory of RCD+CAT spaces}

In this section we study metric measure spaces $(X, d, m)$ satisfying

$$
(X, d, m) \text { is } C A T(\kappa) \text { and satisfies the conditions } R C D(K, N) \text { for } 1 \leq N<\infty, K, \kappa<\infty \text {. }
$$

The following result was proved in [24]

Theorem 3.1 ([24]). Let $(X, d, m)$ satisfy $C D(K, N)$ for $1 \leq N<\infty, K, \kappa \in \mathbb{R}$. Then $X$ is infinitesimally Hilbertian. In particular, $(X, d, m)$ satisfies $R C D(K, N)$.

Remark 3.2. It was shown in [24] that the above theorem also holds if the $C D(K, N)$ assumption in (3.1) is replaced by $C D^{\star}(K, N)$ or $C D^{e}(K, N)$ conditions (see [24] for the definitions). Moreover, in a recent paper [30] Di Marino, Gigli, Pasqualetto and Soultanis show that a $C A T(\kappa)$ space with any Radon measure is infinitesimally Hilbertian. For these reasons (3.1) is equivalent to assuming that $X$ is $C A T(\kappa)$ and satisfies one of the assumptions $C D(K, N), C D^{\star}(K, N)$ or $C D^{e}(K, N)$ with $1 \leq N<\infty, K, \kappa<\infty$.

In [24] we also established the following property of spaces satisfying (3.1):

Proposition 3.3 ([24]). Let $X$ satisfy (3.1). Then $X$ is non-branching.

Next we prove

Proposition 3.4. Let $X$ satisfy (3.1). Then for almost all $p \in X$ it holds that $T_{p}^{g} X \cong \mathbb{R}^{k}$ for some $k \leq N$.

Remark 3.5. Note that from the fact that $X$ is an $R C D$ space it follows that $T_{p} X$ is an Euclidean space for almost all $p \in X$ [21]. However, at this point in the proof we don't know if $T_{p} X \cong T_{p}^{g} X$ at all such points (we expect this to be true for all $p$ ).

Proof. First, recall that by the $C A T$ condition, geodesics of length less than $\pi_{\kappa}$ in $X$ are unique. Moreover, since $X$ is nonbranching and $C D$, for any $p \in X$ the set $E_{p}$ of points $q$, such that the geodesic which connects $p$ and $q$ is not extendible, has measure zero (Remark 2.5).

Let $A=\left\{p_{i}\right\}_{i=1}^{\infty}$ be a countable dense set of points in $X$, and let $C=\bigcup_{i \in \mathbb{N}} E_{p_{i}}$. For any $q \in X \backslash C$ and any $i$ with $d\left(p_{i}, q\right)<\pi_{\kappa}$ the geodesic $\left[p_{i} q\right]$ can be extended slightly past $q$. Since $A$ is dense this implies that for any $q \in X \backslash C$ there is a dense subset in $T_{q}^{g} X$ consisting of directions $v$ which have "opposites" (i.e. making angle $\pi$ with $v$ ). 
For every $p \in X$ and every tangent cone $T_{p} X$ the geodesic tangent cone $T_{p}^{g} X$ is naturally a closed convex subset of $T_{p} X$. Since $X$ is $R C D$ this means that for almost all $p$ the geodesic tangent cone $T_{p}^{g} X$ is a convex subset of a Euclidean space. Thus, for almost all $p \in X$ it holds that $T_{p}^{g} X$ is a convex subset in $\mathbb{R}^{m}$ for some $m \leq N$, is a metric cone over $\Sigma_{p}^{g} X$ and contains a dense subset of points with opposites also in $T_{p}^{g} X$. In particular, $\Sigma_{p}^{g} X$ is a convex subset of $\mathbb{S}^{m}$. Since a closed convex subset of $\mathbb{S}^{m}$ is either $\mathbb{S}^{k}$ with $k \leq m$ or has boundary this means that for any such $p T_{p}^{g} X$ is isometric to a Euclidean space of dimension $k \leq m$.

Proposition 3.6. Let $X$ satisfy (3.1).

i) Let $p \in X$ satisfy $T_{p}^{g} X \cong \mathbb{R}^{m}$ for some $m \leq N$.

Then an open neighbourhood $W$ of $p$ is homeomorphic to $\mathbb{R}^{m}$.

ii) If an open neighborhood $W$ of $p$ is homeomorphic to $\mathbb{R}^{m}$ then for any $q \in W$ it holds that $T_{q}^{g} X \cong T_{q} X \cong \mathbb{R}^{m}$. Moreover, for any compact set $C \subset W$ there is $\varepsilon=\varepsilon(C)>0$ such that every geodesic starting in $C$ can be extended to length at least $\varepsilon$.

Proof. Let us first prove part i). Suppose $T_{p}^{g} X \cong \mathbb{R}^{m}$. By [26, Theorem A] there is a small $R>0$ such that $B_{R}(p) \backslash\{p\}$ is homotopy equivalent to $\mathbb{S}^{m-1}$. Since $\mathbb{S}^{m-1}$ is not contractible, by [28, Theorem 1.5] there is $0<$ $\varepsilon<\pi_{\kappa} / 2$ such that every geodesic starting at $p$ extends to a geodesic of length $\varepsilon$. The natural "logarithm" map $\Phi: \bar{B}_{\varepsilon}(p) \rightarrow \bar{B}_{\varepsilon}(0) \subset T_{p}^{g} X$ is Lipschitz since $X$ is $C A T(\kappa)$. By the above mentioned result of Lytchak and Schroeder [28, Theorem 1.5] $\Phi$ is onto.

We also claim that $\Phi$ is 1-1. If $\Phi$ is not 1-1 then there exist two distinct unit speed geodesics $y_{1}, y_{2}$ of the same length $\varepsilon^{\prime} \leq \varepsilon$ such that $p=y_{1}(0)=y_{2}(0), y_{1}^{\prime}(0)=y_{2}^{\prime}(0)$ but $y_{1}\left(\varepsilon^{\prime}\right) \neq y_{2}\left(\varepsilon^{\prime}\right)$.

Let $v=y_{1}^{\prime}(0)=y_{2}^{\prime}(0)$. Since $T_{p}^{g} X \cong \mathbb{R}^{m}$ the space of directions $T_{p}^{g} X$ contains the "opposite" vector $-v$. Then there is a geodesic $y_{3}$ of length $\varepsilon$ starting at $p$ in the direction $-v$. Since $X$ is $C A T(\kappa)$ and $2 \varepsilon<\pi_{k}$, the concatenation of $y_{3}$ with $y_{1}$ is a geodesic and the same is true for $y_{2}$. This contradicts the fact that $X$ is nonbranching.

Thus, $\Phi$ is a continuous bijection and since both $\bar{B}_{\varepsilon}(p)$ and $\bar{B}_{\varepsilon}(0)$ are compact and Hausdorff it's a homeomorphism. This proves part i).

Let us now prove part ii). Suppose an open neighborhood $W$ of $p$ is homeomorphic to $\mathbb{R}^{m}$.

By [24, Lemma 3.1] or by the same argument as above using [26] and [28], for any $q \in W$ all geodesics starting at $q$ can be extended to length at least $\varepsilon(q)>0$. Therefore $T_{q}^{g} X \cong T_{q} X$. By the splitting theorem $T_{q} X \cong \mathbb{R}^{l}$ where $l=l(q) \leq N$ might a priori depend on $q$. However, using part i) we conclude that an open neighbourhood of $q$ is homeomorphic to $\mathbb{R}^{l(q)}$. Since $W$ is homeomorphic to $\mathbb{R}^{m}$ this can only happen if $l(q)=m$.

The last part of ii) immediately follows from above and compactness of $C$.

\subsection{DC coordinates in $R C D+C A T$ spaces.}

Let $X_{\text {reg }}^{g}$ be the set of points $p$ in $X$ with $T_{p} X \cong T_{p}^{g} X \cong \mathbb{R}^{n}$. Then by Proposition 3.6 there is an open neighbourhood $\hat{U}$ of $p$ homeomorphic to $\mathbb{R}^{n}$ such that every $q \in \hat{U}$ also lies in $X_{\text {reg. }}^{g}$. In particular, $X_{\text {reg }}^{g}$ is open. Further, geodesics in $\hat{U}$ are locally extendible by Proposition 3.6.

Thus the theory of Lytchak-Nagano from [27] applies, and let $x: U \rightarrow V$ with $U=B_{2 \epsilon}(p) \subset \hat{U}$ be $D C$ coordinates as in Subsection 2.5. The pushforward of the Hausdorff measure $\mathcal{H}^{n}$ on $U$ under $x$ coordinates is given by $\sqrt{|g|} \mathcal{L}^{n}$ where $|g|$ is the determinant of $g_{i j}$. Consequently, the map $x:\left(U, d, \mathcal{H}^{n}\right) \rightarrow\left(V, d_{g}, \sqrt{|g|} \mathcal{L}^{n}\right)$ is a metric-measure isomorphism.

With a slight abuse of notations we will identify these metric-measure spaces as well as functions on them, i.e we will identify any function $u$ on $U$ with $u \circ x^{-1}$ on $V$.

Lemma 3.7. Angles between geodesics in $U$ are continuous. That is if $q_{i} \rightarrow q \in U,\left[q_{i} s_{i}\right] \rightarrow[q s],\left[q_{i} t_{i}\right] \rightarrow[q t]$ are converging sequences with $q \neq s, q \neq t$ then $\angle s_{i} q_{i} t_{i} \rightarrow \angle s q t$. 
Proof. Without loss of generality we can assume that $q_{i} \in U$ for all $i$. Let $\alpha_{i}=\angle s_{i} q_{i} t_{i}, \alpha=\angle$ sqt. Let $\left\{\alpha_{i_{k}}\right\}$ be a converging subsequence and let $\bar{\alpha}=\lim _{k \rightarrow \infty} \alpha_{i_{k}}$. Then by upper semicontinuity of angles in $\operatorname{CAT}(\kappa)$ spaces it holds that $\alpha \geq \bar{\alpha}$. We claim that $\alpha=\bar{\alpha}$.

By Proposition 3.6 we can extend $\left[s_{i} q_{i}\right.$ ] past $q_{i}$ as geodesics a definite amount $\delta$ to geodesics $\left[s_{i} z_{i}\right]$. Let $\beta_{i}=\angle z_{i} q_{i} t_{i}$. By possibly passing to a subsequence of $\left\{i_{k}\right\}$ we can assume that $\left[s_{i_{k}} z_{i_{k}}\right] \rightarrow[s z]$. Let $\beta=\angle z q t$. Then since all spaces of directions $T_{q_{i}}^{g} X$ and $T_{q}^{g} X$ are Euclidean by Proposition 3.6, we have that $\alpha_{i}+\beta_{i}=$ $\alpha+\beta=\pi$ for all $i$. Again using semicontinuity of angles we get that $\beta \geq \bar{\beta}$.

We therefore have

$$
\pi=\alpha+\beta \geq \bar{\alpha}+\bar{\beta}=\pi
$$

Hence all the inequalities above are equalities and $\alpha=\bar{\alpha}$. Since this holds for an arbitrary converging subsequence $\left\{\alpha_{i_{k}}\right\}$ it follows that $\lim _{i \rightarrow \infty} \alpha_{i}=\alpha$.

Let $\widetilde{\mathcal{A}}$ be the algebra of functions of the form $\varphi\left(f_{1}, \ldots, f_{m}\right)$ where $f_{i}=d\left(\cdot, q_{i}\right)$ for some $q_{1}, \ldots, q_{m}$ with $\left|q_{i} p\right|>$ $\varepsilon$ and $\varphi$ is smooth. Together with the first variation formula for distance functions Lemma 3.7 implies that for any $u, h \in \widetilde{\mathcal{A}}$ it holds that $\langle\nabla u, \nabla h\rangle_{g}$ is continuous on $V$. In particular, $g^{i j}=\left\langle\nabla x_{i}, \nabla x_{j}\right\rangle_{g}$ is continuous and hence $g$ is $B V_{0}$ and not just BV.

Furthermore, since $\frac{\partial}{\partial x_{i}}=\sum_{j} g_{i j} \nabla x_{j}$ where $g_{i j}$ is the pointwise inverse of $g^{i j}$, Lemma 3.7 also implies that any $u \in \widetilde{\mathcal{A}}$ is $C^{1}$ on $V$. Hence, any such $u$ is $D C_{0}$ on $V$.

Recall that for a Lipschitz function $u$ on $V$ we have two a-priori different notions of the norm of the gradient defined $m$-a.e.: the "Riemannian" norm of the gradient $|\nabla u|_{g}^{2}=g^{i j} \frac{\partial u}{\partial x^{i}} \frac{\partial u}{\partial x_{j}}$ and the minimal weak upper gradient $|\nabla u|$ when $u$ is viewed as a Sobolev function in $W^{1,2}(m)$. We observe that these two notions are equivalent.

Lemma 3.8. Let $u, h: U \rightarrow \mathbb{R}$ be Lipschitz functions. Then $|\nabla u|=|\nabla u|_{g},|\nabla h|=|\nabla h|_{g}$ m-a.e. and $\langle\nabla u, \nabla h\rangle=$ $\langle\nabla u, \nabla h\rangle_{g}$ m-a.e..

In particular, $g^{i j}=\left\langle\nabla x_{i}, \nabla x_{j}\right\rangle_{g}=\left\langle\nabla x_{i}, \nabla x_{j}\right\rangle$ m-a.e.

Proof. First note that since both $\langle\nabla u, \nabla h\rangle$ and $\langle\nabla u, \nabla h\rangle_{g}$ satisfy the parallelogram rule, it's enough to prove that $|\nabla u|=|\nabla u|_{g}$ a.e..

Recall that $g^{i j}$ is continuous on $U$. Fix a point $p$ where $u$ is differentiable. Then

$$
\begin{aligned}
\operatorname{Lip} u(p)=\limsup _{q \rightarrow p} \frac{|u(p)-u(q)|}{d(p, q)} & =\limsup _{q \rightarrow p} \frac{|u(p)-u(q)|}{|p-q|_{g(p)}} \\
& =\sup _{|v|_{g(p)=1}} D_{v} u=\sup _{|v|_{g(p)=1}}\langle v, \nabla u\rangle_{g(p)}=|\nabla u|_{g(p)} .
\end{aligned}
$$

In the second equality we used that $d$ is induced by $g^{i j}$, and that $g^{i j}$ is continuous. Since $(U, d, m)$ admits a local 1-1 Poincaré inequality and is doubling, the claim follows from [14] where it is proved that for such spaces $\operatorname{Lip} u=|\nabla u|$ a.e..

In view of the above Lemma from now on we will not distinguish between $|\nabla u|$ and $|\nabla u|_{g}$ and between $\langle\nabla u, \nabla h\rangle$ and $\langle\nabla u, \nabla h\rangle_{g}$.

Proposition 3.9. If $u \in W^{1,2}(m) \cap B V(U)$, then $|\nabla u|^{2}=g^{i j} \frac{\partial^{a p} u}{\partial x_{i}} \frac{\partial^{a p} u}{\partial x_{j}} m$-a.e. .

Proof. We choose a set $S \subset U$ of full measure such that $u$ and $|\nabla u|$ are defined pointwise on $S$ and $u$ is approximately differentiable at every $x \in S$. Since $u$ is $B V(U)$, for $\eta>0$ there exist $\hat{u}_{\eta} \in C^{1}(U)$ such that for the set

$$
B_{\eta}=\left\{x \in S: u(x) \neq \hat{u}_{\eta}(x), D^{a p} u(x) \neq D \hat{u}(x)\right\} \cap S
$$

one has $m\left(B_{\eta}\right) \leq \eta$ [17, Theorem 6.13]. Note, since $f$ is continuous, there exists a constant $\lambda>0$ such that $\lambda^{-1} m \leq \mathcal{H}^{n} \leq \lambda m$ on $U$. Moreover, since $g^{i j}$ is continuous, one can check that $\hat{u}_{\eta}$ is Lipschitz w.r.t. $d_{g}$, and hence $\hat{u}_{\eta} \in W^{1,2}(m)$. 
By [3, Proposition 4.8] we know that $\left|\nabla u\left\|_{A_{\eta}}=\mid \nabla \hat{u}_{\eta}\right\|_{A_{\eta}} m\right.$-a.e. for $A_{\eta}=S \backslash B_{\eta}$. On the other hand, uniqueness of approximative derivatives also yields that $\left.g^{i j} \frac{\partial^{a p} u}{\partial x_{i}} \frac{\partial^{a p} u}{\partial x_{j}}\right|_{A_{\eta}}=\left.g^{i j} \frac{\partial^{a p} \hat{u}_{\eta}}{\partial x_{i}} \frac{\partial^{a p} \hat{u}_{\eta}}{\partial x_{j}}\right|_{A_{\eta}} m$-a.e. . Hence, since $\hat{u}$ is Lipschitz w.r.t. $d$,

$$
|\nabla u| 1_{A_{\eta}}=g^{i j} \frac{\partial^{a p} u}{\partial x_{i}} \frac{\partial^{a p} u}{\partial x_{j}} 1_{A_{\eta}} m \text {-a.e. . }
$$

by Lemma 3.8.

Now, we pick a sequence $\eta_{k}$ for $k \in \mathbb{N}$ such that $\sum_{k=1}^{\infty} \eta_{k}<\infty$. Then, by the Borel-Cantelli Lemma the set

$$
B=\left\{x \in S: \exists \text { infinitely many } k \in \mathbb{N} \text { s.t. } x \in B_{\eta_{k}}\right\}
$$

is of $m$-measure 0 . Consequently, for $x \in A=S \backslash B$ we can pick a $k \in \mathbb{N}$ such that $x \in A_{\eta_{k}} \subset S$. It follows

$$
|\nabla u|^{2}(x)=g^{i j} \frac{\partial^{a p} u}{\partial x_{i}} \frac{\partial^{a p} u}{\partial x_{j}}(x) \forall x \in S
$$

and hence $m$-a.e. .

\section{Proof of the main theorem}

0. Let $\left(X, d, f \mathcal{H}^{n}\right)$ be $R C D(K, n)$ and $C A T(\kappa)$ where $0 \leq f \in L_{l o c}^{1}\left(\mathcal{H}^{n}\right)$.

Remark 4.1. If $(X, d, m)$ is a weakly non-collapsed $R C D$ space in the sense of [16] or a space satisfying the generalized Bishop inequality in the sense of [23] and if $(X, d)$ is $C A T(\kappa)$, the assumptions are satisfied by [16, Theorem 1.10].

Following Gigli and De Philippis [16] for any $x \in X$ we consider the monotone quantity $\frac{m\left(B_{r}(x)\right)}{v_{k, n}(r)}$ which is non increasing in $r$ by the Bishop-Gromov volume comparison. Let $\theta_{n, r}(x)=\frac{m\left(B_{r}(x)\right)}{\omega_{n} r^{n}}$. Consider the density function $\theta_{n}(x)=\lim _{r \rightarrow 0} \theta_{n, r}(x)=\lim _{r \rightarrow 0} \frac{m\left(B_{r}(x)\right)}{\omega_{n} r^{n}}$.

Since $n$ is fixed throughout the proof we will drop the subscripts $n$ and from now on use the notations $\theta(x)$ and $\theta_{r}(x)$ for $\theta_{n}(x)$ and $\theta_{n, r}(x)$ respectively.

By Propositions 3.4, 3.6 and [16, Theorem 1.10] we have that for almost all $p \in X$ it holds that $T_{p} X \cong$ $T_{p}^{g} X \cong \mathbb{R}^{n}$ and $\theta(x)=f(x)$.

Therefore we can and will assume from now on that $f=\theta$ everywhere.

Remark 4.2. Monotonicity of $r \mapsto \frac{m\left(B_{r}(x)\right)}{v_{k, n}(r)}$ immediately implies that $f(x)=\theta(x)>0$ for all $x$.

Let $x \in X_{\text {reg }}^{g}$. Then $T_{p}^{g} X \cong \mathbb{R}^{l}$ for some $l \leq n$. We claim that $l=n$. By Proposition $3.6 X_{\text {reg }}^{g}$ is an $l$-manifold near $p$ and by section 2.5 DC coordinates near $p$ give a biLipschitz homeomorphism of an open neighborhood of $p$ onto an open set in $\mathbb{R}^{m}$. Since $m=f \mathcal{H}^{n}$ this can only happen if $l=n$.

Lemma 4.3. [24, Lemma 5.4] $\theta^{\frac{1}{n}}=f^{\frac{1}{n}}$ is semiconcave on $X$.

Corollary 4.4. $\theta=f$ is locally Lipschitz near any $p \in X_{\text {reg. }}^{g}$.

Proof. First observe that semiconcavity of $\theta^{\frac{1}{n}}$, the fact that $\theta>0$ and local extendability of geodesics on $X_{\text {reg }}^{g}$ imply that $\theta$ must be locally bounded on $X_{r e g}^{g}$. Now the corollary becomes an easy consequence of Lemma 4.3, the fact that geodesics are locally extendible a definite amount near $p$ by Proposition 3.6 and the fact that a semiconcave function on $(0,1)$ is locally Lipschitz.

1. Since small balls in spaces with curvature bounded above are geodesically convex, we can assume that $\operatorname{diam} X<\pi_{\kappa}$. Let $p \in X, x: U \rightarrow \mathbb{R}^{n}$ and $\widetilde{\mathcal{A}}$ be as in the previous subsection.

By the same argument as in [32, Section 4] (cf. [33], [1]) it follows that any $u \in \widetilde{\mathcal{A}}$ lies in $D\left(\Delta, U, \mathcal{H}^{n}\right)$ and the $\mathcal{H}^{n}$-absolutely continuous part of $\Delta_{0} u$ can be computed using standard Riemannian geometry formulas that 
is

$$
\Delta_{0}^{a}(u)=\frac{1}{\sqrt{|g|}} \frac{\partial^{a p}}{\partial x_{j}}\left(g^{j k} \sqrt{|g|} \frac{\partial u}{\partial x_{k}}\right)
$$

where $|g|$ denotes the pointwise determinant of $g^{i j}$. Here $\Delta_{0}$ denotes the measure valued Laplacian on $\left(U, d, \mathcal{H}^{n}\right)$. Note that $g, \sqrt{|g|}$ and $\frac{\partial u}{\partial x_{i}}$ are $B V_{0}$ functions, and the derivatives on the right are understood as approximate derivatives.

Indeed, w.l.o.g. let $u \in D C_{0}(U)$, and let $v$ be Lipschitz with compact support in $U$. As before we identify $u$ and $v$ with their representatives in $x$ coordinates. First, we note that, since $g, \sqrt{|g|}$ and $\frac{\partial u}{\partial x_{i}}$ are $B V_{0}$, their product is also in $B V_{0}$, as well as the product with $v$. Then, the Leibniz rule (2.3) for the approximate partial derivatives yieds that

$$
\sqrt{|g|} g^{i j} \frac{\partial u}{\partial x_{i}} \frac{\partial^{a p} v}{\partial x_{j}}=-\frac{\partial^{a p}}{\partial x_{j}}\left(\sqrt{|g|} g^{i j} \frac{\partial u}{\partial x_{i}}\right) v+\frac{\partial^{a p}}{\partial x_{j}}\left(\sqrt{|g|} g^{i j} \frac{\partial u}{\partial x_{i}} v\right) \mathcal{L}^{n} \text {-a.e. . }
$$

Again using (2.3) we also have that

$$
\sqrt{|g|} g^{i j} \frac{\partial u}{\partial x_{i}} \frac{\partial v}{\partial x_{j}}=-\frac{\partial}{\partial x_{j}}\left(\sqrt{|g|} g^{i j} \frac{\partial u}{\partial x_{i}}\right) v+\frac{\partial}{\partial x_{j}}\left(\sqrt{|g|} g^{i j} \frac{\partial u}{\partial x_{i}} v\right) \text { as measures }
$$

and the absolutely continuous with respect to $\mathcal{L}^{n}$ part of this equation is given by the previous identity.

The fundamental theorem of calculus for BV functions (see [17, Theorem 5.6]) yields that

$$
\int_{V} \frac{\partial}{\partial x_{j}}\left(\sqrt{|g|} g^{i j} \frac{\partial u}{\partial x_{i}} v\right)=0 .
$$

Moreover, by Lemma $3.8\langle\nabla v, \nabla u\rangle$ is given in $x$ coordinates by $g^{i j} \frac{\partial v}{\partial x_{j}} \frac{\partial u}{\partial x_{i}} \mathcal{L}^{n}$-a.e. .

Combining the above formulas gives that

$$
\begin{aligned}
-\int_{V}\langle\nabla u, \nabla v\rangle \sqrt{|g|} d \mathcal{L}^{n} & =\int_{V}\left[\frac{\partial^{a p}}{\partial x_{j}}\left(\sqrt{|g|} g^{i j} \frac{\partial u}{\partial x_{i}}\right) v-\frac{\partial^{a p}}{\partial x_{j}}\left(\sqrt{|g|} g^{i j} \frac{\partial u}{\partial x_{i}} v\right)\right] d \mathcal{L}^{n} \\
& =\int_{V} \frac{1}{\sqrt{|g|}} \frac{\partial^{a p}}{\partial x_{j}}\left(\sqrt{|g|} g^{i j} \frac{\partial u}{\partial x_{i}}\right) v \sqrt{|g|} d \mathcal{L}^{n}+\int_{V} v d \mu
\end{aligned}
$$

where $\mu$ is some signed measure such that $\mu \perp \mathcal{L}^{n}$. This implies (4.1).

2. Since $(X, d, m)$ is $R C D(K, n)$ for any $q \in X$, we have that $d_{q}$ lies in $D(\Delta, U \backslash\{q\}, m)$ and $\Delta d_{q}$ is locally bounded above on $U \backslash\{q\}$ by const $\cdot m$ by Theorem 2.4 .

Furthermore, since by Proposition 3.6 all geodesics in $U$ are locally extendible we have $\Delta d_{q}=\left[\Delta d_{q}\right]^{r e g} \cdot \mathrm{m}$ on $U \backslash\{q\}$ and $\left[\Delta d_{q}\right]^{\text {reg }}$ is locally bounded below on $U \backslash\{q\}$ again by Theorem 2.4. Therefore $\left[\Delta d_{q}\right]^{\text {reg }}$ is in $L_{\text {loc }}^{\infty}\left(U \backslash\{q\}\right.$ ) with respect to $m$ (and also $\mathcal{H}^{n}$ ), and in particular, $\Delta d_{q}$ is locally $L^{2}$ in $U \backslash\{q\}$.

By the chain rule for $\Delta[19]$ the same holds for any $u, h \in \widetilde{\mathcal{A}}$ on all of $U$ as by construction $u$ and $h$ only involve distance functions to points outside $U$.

Recall the following lemma from [7, Lemma 6.7] (see also [31]).

Lemma 4.5. Let $(X, d, m)$ be a metric measure space satisfying a $R C D$ condition. Then for all $E \subset X$ compact and all $G \subset X$ open such that $E \subset G$ there exists a Lipschitz function $\chi: X \rightarrow[0,1]$ with

(i) $\chi=1$ on $E_{v}=\{x \in X: \exists y \in E: d(x, y)<v\}$ and $\operatorname{supp} \chi \subset G$,

(ii) $\Delta \chi \in L^{\infty}(m)$ and $|\nabla \chi|^{2} \in W^{1,2}(X)$.

Let us choose a cut-off function $\chi: X \rightarrow[0,1]$ as in the previous lemma for $G$ with $\bar{G} \subset U$ and $E=\bar{B}_{\delta}(p) \subset G$ for some $\delta \in(0, \varepsilon)$. 
Let $u, h \in \widetilde{\mathcal{A}}$. By the chain rule for $\Delta$ it again follows that

$$
\boldsymbol{\Delta}(\chi u)=[\boldsymbol{\Delta}(\chi u)]^{r e g} m \&[\boldsymbol{\Delta}(\chi u)]^{r e g} \in L^{2}(m) .
$$

Moreover, (2.1) holds for Lipschitz functions on $X$. Hence $\chi u \in D_{L^{2}(m)}(\Delta)$.

Therefore $\chi u, \chi h \in H^{2,2}(X)$ by Remark 2.8, $\langle\nabla \chi u, \nabla \chi h\rangle \in W^{1,2}(U)$ by Proposition 2.9 and the Hessian of $\chi u$ can be computed by the formula (2.2). Moreover, by locality of the minimal weak upper gradient

$$
\begin{aligned}
& \left.2 \operatorname{Hess}(\chi u)\left(\nabla\left(\chi h_{1}\right), \nabla\left(\chi h_{2}\right)\right)\right|_{B_{\delta}(p)} \\
& \quad=\left\langle\nabla h_{1}, \nabla\left\langle\nabla u, \nabla h_{2}\right\rangle\right\rangle+\left\langle\nabla h_{2}, \nabla\left\langle\nabla u, \nabla h_{1}\right\rangle\right\rangle-\left\langle\nabla u, \nabla\left\langle\nabla h_{1}, \nabla h_{2}\right\rangle\right\rangle m \text {-a.e. in } \bar{B}_{\delta}(p) .
\end{aligned}
$$

Note that, for instance,

$$
\left.W^{1,2}\left(\bar{B}_{\delta}(p)\right) \ni\left\langle\nabla \chi u, \nabla \chi h_{2}\right\rangle\right|_{\bar{B}_{\delta}(p)}=\left\langle\left.\nabla \chi u\right|_{\bar{B}_{\delta}(p)},\left.\nabla \chi h_{2}\right|_{\bar{B}_{\delta}(p)}\right\rangle=\left\langle\left.\nabla u\right|_{\bar{B}_{\delta}(p)},\left.\nabla h_{2}\right|_{\bar{B}_{\delta}(p)}\right\rangle .
$$

Remark 4.6. It is not clear that $u$ itself is in the domain of Gigli's Hessian since $u$ is not contained $D_{L^{2}(m)}(\Delta)$ (integration by parts for $u$ would involve boundary terms). Nevertheless, the equality and the RHS in (4.4) are well-defined on $B_{\delta}(p)$. We denote the RHS in (4.4) with $H u\left(h_{1}, h_{2}\right)$.

3. The aim of this paragraph is to compute $H u\left(x_{i}, x_{j}\right) g_{i j}$ on $B_{\delta}(p)$ in the $D C_{0}$ coordinate chart $x$. In the following we assume w.l.o.g. that $B_{\varepsilon}(p)=B_{\delta}(p)$ for $\delta$ like in the previous paragraph.

Since $u, h_{1}, h_{2}$ are $D C_{0}$ in $x$ coordinates we have that $\left\langle\nabla h_{1}, \nabla h_{2}\right\rangle=g^{i j} \frac{\partial h_{1}}{\partial x_{i}} \frac{\partial h_{2}}{\partial x_{j}}$ is $B V$ and the same holds for $\left\langle\nabla u, \nabla h_{1}\right\rangle,\left\langle\nabla u, \nabla h_{2}\right\rangle$. Moreover, $\left\langle\nabla h_{i}, \nabla h_{j}\right\rangle,\left\langle\nabla u, \nabla h_{i}\right\rangle \in W^{1,2}\left(\bar{B}_{\delta}(p)\right)$ as we saw before.

Hence, with the help of Proposition 3.9 the RHS of (4.4) can be computed pointwise in $x$ coordinates at points of approximate differentiability of $\frac{\partial u}{\partial x_{i}}, \frac{\partial h_{1}}{\partial x_{i}}$ and $\frac{\partial h_{2}}{\partial x_{i}}, i=1, \ldots n$, and (4.4) can be understood to hold a.e. in the sense of approximate derivatives. That is, we can write

$$
\left\langle\nabla u, \nabla\left\langle\nabla h_{1}, \nabla h_{2}\right\rangle\right\rangle=g^{i j} \frac{\partial^{a p} u}{\partial x_{i}} \frac{\partial^{a p}}{\partial x_{j}}\left\langle\nabla h_{1}, \nabla h_{2}\right\rangle=g^{i j} \frac{\partial u}{\partial x_{i}} \frac{\partial^{a p}}{\partial x_{j}}\left(g^{k l} \frac{\partial h_{1}}{\partial x_{k}} \frac{\partial h_{2}}{\partial x_{l}}\right)
$$

and do the same for the other two terms in the RHS of (4.4). that

Using that $g^{i j}=\left\langle\nabla x_{i}, \nabla x_{j}\right\rangle$ and $\frac{\partial}{\partial x_{i}}=\sum_{j} g_{i j} \nabla x_{j}$ a standard computation shows that for any $u \in \widetilde{\mathcal{A}}$ it holds

$$
\frac{1}{\sqrt{|g|}} \frac{\partial^{a p}}{\partial x_{j}}\left(g^{j k} \sqrt{|g|} \frac{\partial u}{\partial x_{k}}\right)=H u\left(x_{i}, x_{j}\right) g_{i j}
$$

on $B_{\delta}(p)$.

Let $S$ be the set of points in $V$ where $\nabla u, g_{i j}$ have approximate derivatives and $\frac{\partial^{a p}}{\partial x_{i}}\left(\frac{\partial u}{\partial x_{j}}\right)=\frac{\partial^{a p}}{\partial x_{j}}\left(\frac{\partial u}{\partial x_{i}}\right)$. Then by (2.4) $S$ has full measure in $V$, and hence it's enough to verify (4.6) pointwise on $S$.

Let $q \in S$. Let $\hat{g}$ be a smooth metric on a neighborhood of $q$ which such that $\hat{g}(q)=g(q)$ and $D \hat{g}(q)=$ $D^{a p} g(q)$. Likewise let $\hat{u}$ be a smooth function on a neighborhood of $q$ such that $\hat{u}(q)=u(q), D \hat{u}(q)=D u(q)$ and $D \frac{\partial \hat{u}}{\partial x_{i}}(q)=D^{a p} \frac{\partial u}{\partial x_{i}}(q)$ for all $i$. Such $\hat{u}$ exists (we can take it to be quadratic in $x$ ) since $\frac{\partial^{a p}}{\partial x_{i}}\left(\frac{\partial u}{\partial x_{i}}\right)(q)=\frac{\partial^{a p}}{\partial x_{j}}\left(\frac{\partial u}{\partial x_{i}}\right)(q)$. Then

$$
\frac{1}{\sqrt{|g|}} \frac{\partial^{a p}}{\partial x_{j}}\left(g^{j k} \sqrt{|g|} \frac{\partial u}{\partial x_{k}}\right)(q)=\frac{1}{\sqrt{|\hat{g}|}} \frac{\partial}{\partial x_{j}}\left(\hat{g}^{j k} \sqrt{|\hat{g}|} \frac{\partial \hat{u}}{\partial x_{k}}\right)(q)
$$

where all the derivatives are approximate derivatives.

Similarly

$$
H u\left(x_{i}, x_{j}\right)(q) g_{i j}(q)=H \hat{u}\left(x_{i}, x_{j}\right)(q) \hat{g}_{i j}(q)
$$

where again all the derivatives in (4.4) and (4.5) are approximate derivatives.

But

$$
\frac{1}{\sqrt{|\hat{g}|}} \frac{\partial}{\partial x_{j}}\left(\hat{g}^{j k} \sqrt{|\hat{g}|} \frac{\partial \hat{u}}{\partial x_{k}}\right)(q)=\operatorname{Hess}_{\hat{g}} \hat{u}\left(\nabla_{\hat{g}} x_{i}, \nabla_{\hat{g}} x_{j}\right) \hat{g}_{i j}(q)
$$

by standard Riemannian geometry since all functions involved are smooth. Since $q \in S$ was arbitrary this proves that (4.6) holds a.e. in the sense of approximate derivatives as claimed. 
4. It follows that

$$
\begin{aligned}
& \left.\operatorname{Tr} \operatorname{Hess}(\chi u)\right|_{B_{\delta}(p)}=\left.\operatorname{Hess}(\chi u)\left(\nabla x_{i}, \nabla x_{j}\right) g_{i j}\right|_{B_{\delta}(p)} \\
& \quad=\left.\operatorname{Hess}(\chi u)\left(\nabla \chi x_{i}, \nabla \chi x_{j}\right) g_{i j}\right|_{B_{\delta}(p)}=H u\left(x_{i}, x_{j}\right) g_{i j}=\left.\Delta_{0} u\right|_{B_{\delta}(p)}
\end{aligned}
$$

for every $u \in \widetilde{\mathcal{A}}$ where Hess is the Hessian in the sense of Gigli, and $H(u)$ is denotes the RHS of (4.4). The first equality in (4.7) is the definition of $\operatorname{Tr}$, the second equality is the $L^{\infty}$-homogeneity of the tensor $\operatorname{Hess}(\chi u)$, and the third equality is the identity (4.4).

Since $f$ is locally Lipschitz and positive on $B_{\delta}(p)$, we can perform the following integration by parts in $D C_{0}$ coordinates. Let $u \in \widetilde{\mathcal{A}}$ and let $g$ be a Lipschitz function with compact support in $B_{\delta}(p) \cdot \chi u \in D_{L^{2}(m)}(\Delta)$ implies $\left.u\right|_{B_{\delta}(p)} \in D\left(\Delta, B_{\delta}(p)\right)$. Then

$$
\begin{aligned}
\int_{B_{\delta}(p)} g \Delta u d m & =-\int_{B_{\delta}(p)}\langle\nabla u, \nabla g\rangle d m=-\int_{B_{\delta}(p)}\langle\nabla u, \nabla g\rangle f d \mathcal{H}^{n} \\
& =-\int_{B_{\delta}(p)}\langle\nabla u, \nabla(g f)\rangle d \mathcal{H}^{n}+\int_{B_{\delta}(p)}\langle\nabla u, \nabla \log f\rangle g f d \mathcal{H}^{n} \\
& =\int_{B_{\delta}(p)}\left(\Delta_{0} u+\langle\nabla u, \nabla \log f\rangle\right) g d m
\end{aligned}
$$

yields

$$
\Delta u=\Delta_{0} u+\langle\nabla u, \nabla \log f\rangle
$$

on $B_{\delta}(p)$ for any $u \in \widetilde{\mathcal{A}}$. Note again that only $\chi u$ is in $D_{L^{2}(m)}(\Delta)$.

On the other hand, by Corollary 2.12 it holds that $\Delta(\chi u)=\operatorname{Tr} \operatorname{Hess}(\chi u) m$-a.e. . Thus

$$
0=\left.\operatorname{Tr} \operatorname{Hess}(\chi u)\right|_{B_{\delta}(p)}-\left.\operatorname{Tr} \operatorname{Hess}(\chi u)\right|_{B_{\delta}(p)}=\left.\Delta u\right|_{B_{\delta}(p)}-\left.\Delta_{0}(\chi u)\right|_{B_{\delta}(p)}=\left.\langle\nabla u, \nabla \log f\rangle\right|_{B_{\delta}(p)}
$$

a.e. for any $u \in \widetilde{\mathcal{A}}$.

5. Therefore $\left.f \nabla \log f\right|_{B_{\delta}(p)}=\left.\nabla f\right|_{B_{\delta}(p)}=0$. Indeed, since $f$ is semiconcave, $f \circ x^{-1}$ is DC by [27]. Hence $\nabla f=g^{i j} \frac{\partial f}{\partial x_{i}}$ is continuous on a set of full measure $Z$ in $B_{\delta}(p)$ since this is true for convex functions on $\mathbb{R}^{n}$. Let $q \in Z$ be a point of continuity of $\left.\nabla f\right|_{Z}$ and $v=\nabla f(q)$. Assume $v \neq 0$. Then due to extendability of geodesics there exists $z \notin U$ such that $\nabla d_{z}(q)=\frac{v}{|v|}$. Since $\nabla d_{z}$ is continuous near $q$ and $\nabla f$ is continuous on $Z$ it follows $\left\langle\nabla f, \nabla d_{z}\right\rangle \neq 0$ on a set of positive measure. Hence $\left.\nabla f\right|_{B_{\delta}(p)}=0$ and $\left.f\right|_{B_{\delta}(p)}=$ const.

6. We claim that this implies that $f$ is constant on $X_{\text {reg }}^{g}$. (This is not immediate since we don't know yet that $X_{\text {reg }}^{g}$ is connected.) Indeed, since $X$ is essentially nonbranching, radial disintegration of $m$ centered at $p$ (Theorem 2.4) implies that for almost all $q \in X$ the set $[p q] \cap X_{r e g}^{g}$ has full measure in $[p, q]$. It is also open in $[p, q]$ since $X_{\text {reg }}^{g}$ is open.

Suppose $q \in X_{\text {reg }}^{g}$ is as above.

Since $\theta$ is semiconcave on $X$ and locally constant on $X_{\text {reg }}^{g}$ it is locally Lipschitz (and hence Lipschitz) on the geodesic segment $[p, q]$. A Lipschitz function on $[0,1]$ which is locally constant on an open set of full $\mathcal{L}^{1}$ measure is constant. Therefore $\theta$ is constant on $[p, q]$ and hence $\theta$ is constant on $X_{\text {reg }}^{g}$ which has full measure. Therefore $f=\theta=$ const a.e. globally.

Acknowledgements: The first author is funded by a Discovery grant from NSERC. The second author is funded by the Deutsche Forschungsgemeinschaft (DFG, German Research Foundation) - Projektnummer 396662902. We are grateful to Alexander Lytchak for a number of helpful conversations. 


\section{References}

[1] Luigi Ambrosio and Jérôme Bertrand, DC calculus, Math. Z. 288 (2018), no. 3-4, 1037-1080. MR 3778989

[2] Luigi Ambrosio, Nicola Gigli, and Giuseppe Savaré, Density of Lipschitz functions and equivalence of weak gradients in metric measure spaces, Rev. Mat. Iberoam. 29 (2013), no. 3, 969-996. MR 3090143

[3] Calculus and heat flow in metric measure spaces and applications to spaces with Ricci bounds from below, Invent. Math. 195 (2014), no. 2, 289-391. MR 3152751

[4] _ Metric measure spaces with Riemannian Ricci curvature bounded from below, Duke Math. J. 163 (2014), no. 7, 1405-1490. MR 3205729

[5] Luigi Ambrosio, Nicola Gigli, and Giuseppe Savaré, Bakry-Émery curvature-dimension condition and Riemannian Ricci curvature bounds, Ann. Probab. 43 (2015), no. 1, 339-404. MR 3298475

[6] L. Ambrosio, A. Mondino, and G. Savaré, Nonlinear diffusion equations and curvature conditions in metric measure spaces, ArXiv e-prints (2015).

[7] Luigi Ambrosio, Andrea Mondino, and Giuseppe Savaré, On the Bakry-Émery condition, the gradient estimates and the local-to-global property of $R C D^{\star}(K, N)$ metric measure spaces, J. Geom. Anal. 26 (2016), no. 1, 24-56. MR 3441502

[8] Dominique Bakry, L'hypercontractivité et son utilisation en théorie des semigroupes, Lectures on probability theory (SaintFlour, 1992), Lecture Notes in Math., vol. 1581, Springer, Berlin, 1994, pp. 1-114. MR 1307413 (95m:47075)

[9] Dmitri Burago, Yuri Burago, and Sergei Ivanov, A course in metric geometry, Graduate Studies in Mathematics, vol. 33, American Mathematical Society, Providence, RI, 2001. MR 1835418 (2002e:53053)

[10] Martin R. Bridson and André Haefliger, Metric spaces of non-positive curvature, Grundlehren der Mathematischen Wissenschaften [Fundamental Principles of Mathematical Sciences], vol. 319, Springer-Verlag, Berlin, 1999. MR MR1744486 (2000k:53038)

[11] Elia Brué and Daniele Semola, Constancy of the dimension for $R C D(K, N)$ spaces via regularity of lagrangian flows, Communications on Pure and Applied Mathematics.

[12] Kathrin Bacher and Karl-Theodor Sturm, Localization and tensorization properties of the curvature-dimension condition for metric measure spaces, J. Funct. Anal. 259 (2010), no. 1, 28-56. MR 2610378 (2011i:53050)

[13] Jeff Cheeger and Tobias H. Colding, On the structure of spaces with Ricci curvature bounded below. I, J. Differential Geom. 46 (1997), no. 3, 406-480. MR 1484888 (98k:53044)

[14] Jeff Cheeger, Differentiability of Lipschitz functions on metric measure spaces, Geom. Funct. Anal. 9 (1999), no. 3, 428517. MR 1708448 (2000g:53043)

[15] Fabio Cavalletti and Andrea Mondino, New formulas for the laplacian of distance functions and applications, arXiv:1803.09687, 2018.

[16] Guido De Philippis and Nicola Gigli, Non-collapsed spaces with Ricci curvature bounded from below, J. Éc. polytech. Math. 5 (2018), 613-650. MR 3852263

[17] Lawrence C. Evans and Ronald F. Gariepy, Measure theory and fine properties of functions, revised ed., Textbooks in Mathematics, CRC Press, Boca Raton, FL, 2015. MR 3409135

[18] Matthias Erbar, Kazumasa Kuwada, and Karl-Theodor Sturm, On the equivalence of the entropic curvature-dimension condition and Bochner's inequality on metric measure spaces, Invent. Math. 201 (2015), no. 3, 993-1071. MR 3385639

[19] Nicola Gigli, On the differential structure of metric measure spaces and applications, Mem. Amer. Math. Soc. 236 (2015), no. 1113, vi+91. MR 3381131

[20] N_ Nonsmooth differential geometry-an approach tailored for spaces with Ricci curvature bounded from below, Mem. Amer. Math. Soc. 251 (2018), no. 1196, v+161. MR 3756920

[21] Nicola Gigli, Andrea Mondino, and Tapio Rajala, Euclidean spaces as weak tangents of infinitesimally Hilbertian metric measure spaces with Ricci curvature bounded below, J. Reine Angew. Math. 705 (2015), 233-244. MR 3377394

[22] Bang-Xian Han, Ricci tensor on $\operatorname{RCD}^{\star}(K, N)$ spaces, J. Geom. Anal. 28 (2018), no. 2, 1295-1314. MR 3790501

[23] Yu Kitabeppu, A Bishop-type inequality on metric measure spaces with Ricci curvature bounded below, Proc. Amer. Math. Soc. 145 (2017), no. 7, 3137-3151. MR 3637960

[24] Vitali Kapovitch and Christian Ketterer, CD meets CAT, arXiv:1712.02839, 2018.

[25] Martin Kell and Andrea Mondino, On the volume measure of non-smooth spaces with Ricci curvature bounded below, 2018. MR 3801291

[26] Linus Kramer, On the local structure and the homology of CAT( $\kappa)$ spaces and Euclidean buildings, Adv. Geom. 11 (2011), no. 2, 347-369. MR 2795430

[27] Alexander Lytchak and Koichi Nagano, Geodesically complete spaces with an upper curvature bound, 2019. MR 3925112

[28] Alexander Lytchak and Viktor Schroeder, Affine functions on CAT( $\kappa)$-spaces, Math. Z. 255 (2007), no. 2, 231-244. MR 2262730

[29] John Lott and Cédric Villani, Ricci curvature for metric-measure spaces via optimal transport, Ann. of Math. (2) 169 (2009), no. 3, 903-991. MR 2480619 (2010i:53068)

[30] Simone Di Marino, Nicola Gigli, Enrico Pasqualetto, and Elefterios Soultanis, Infinitesimal hilbertianity of locally 'cat'( $(\kappa)$ spaces, arXiv:1812.02086, 2018. 
[31] Andrea Mondino and Aaron Naber, Structure theory of metric measure spaces with lower Ricci curvature bounds, 2019. MR 3945743

[32] G. Perelman, DC structure on Alexandrov space with curvature bounded below., preprint, http://www.math.psu.edu/petrunin/papers/papers.html, 1995.

[33] Anton Petrunin, Alexandrov meets Lott-Villani-Sturm, Münster J. Math. 4 (2011), 53-64. MR 2869253 (2012m:53087)

[34] Giuseppe Savaré, Self-improvement of the Bakry-Émery condition and Wasserstein contraction of the heat flow in $\operatorname{RCD}(K, \infty)$ metric measure spaces, Discrete Contin. Dyn. Syst. 34 (2014), no. 4, 1641-1661. MR 3121635

[35] Karl-Theodor Sturm, On the geometry of metric measure spaces. II, Acta Math. 196 (2006), no. 1, 133-177. MR 2237207 (2007k:53051b) 